\title{
COMPUTATIONAL STUDIES ON DIFFERENT TYPES OF APOPTOTIC PROTEINS DOCKED WITH A DIETARY FLAVONOID ERIODICTYOL IN COLON CANCER
}

\author{
MARIYAPPAN PALANI, KARTHI NATESAN, MANJU VAIYAPURI*
}

Department of Biochemistry, Periyar University, Salem, Tamil Nadu, India. Email: manjucb11@gmail.com

Received: 31 August 2016, Revised and Accepted: 06 October 2016

\section{ABSTRACT}

Objective: In this study, to evaluate the computational studies of eriodictyol docked with apoptotic proteins.

Methods: AutoDock Vina and Molecular Graphics Laboratory tools were used to determine the interaction between proteins and eriodictyol. Discovery studio visualizer and PyMOL were used to determine the interaction of amino acid residues between apoptotic proteins and eriodictyol.

Results: The obtained results revealed more binding affinities of p53, caspase 8, B-cell lymphoma (Bcl)-2, Bcl-2 - associated X protein (BAX), and adenomatous polyposis coli (APC) of $-10.6,-10.9,-9.0,-9.5$, and $7.2 \mathrm{kcal} / \mathrm{mol}$, respectively. Interaction of hydrophobic polar contacts of amino acid residues of p53 (CYS-277 and ALA-276), caspase 8 [THR-467, THR-337 (2), and GLU-396 (2)], Bcl-2 [ARG-103 (3), ALA-104 (2), and PHE-105, TYR101], BAX [GLY-108, TRY-107, ASN-106, and GLN-155 (2)], and APC [GLU-40 (2) and LEU-37 (2)] were notified between macromolecules and small molecules. The calculation of root-mean-square deviation of proteins and eriodictyol showed the lower binding energies to be 11.6, 12.5, 15.9, 19.6, and 15.8 and the upper binding energies to be $12.4,15.3,16.9,20.7$, and 18 , respectively. The homology of binding energies was determined below $2 \AA$ which is computationally less expensive and easily determined the hydrophobic polar contacts.

Conclusions: The homology of binding energies was determined below $2 \AA$ which is computationally less expensive and easily determined the hydrophobic polar contacts. The results were proved that the eriodictyol highly interacted with apoptotic proteins. It might be a strong anti-inflammatory activity of colon cancer. In future, computational molecular docking studies should aid further in vitro and in vivo studies.

Keywords: AutoDock Vina, p53, Eriodictyol, Caspase 8, B-cell lymphoma-2, Bcl-2 - associated X protein, Adenomatous polyposis coli.

(C) 2017 The Authors. Published by Innovare Academic Sciences Pvt Ltd. This is an open access article under the CC BY license (http://creativecommons. org/licenses/by/4. 0/) DOI: http://dx.doi.org/10.22159/ajpcr.2017.v10i1.14982

\section{INTRODUCTION}

Colorectal adenocarcinoma is one of the leading causes of morbidity and mortality in the Western world [1]. Currently, an urgent need exists for the discovery and development of novel preventive and therapeutic strategies to combat the severity of the colon cancer. The American Cancer Society has estimated the epidemiological data of colon cancer to be 95,270 new cases and 49,190 deaths in 2016 [2]. In colon cancer treatments, the surviving drugs are proving in inhibiting the molecular targets, particularly by novel agents [3]. Eriodictyol is a strong flavonoid extracted from Eriodictyon californicium and is also called as California yerba santa [4]. It exhibits well-known antioxidant, anti-inflammatory, antineoplastic, antimicrobial, and antinociception activities (Fig.1) [5-8].

Mitochondria, the powerhouse of the cell, play a key role in the regulation of apoptosis and signaling and cellular differentiation. In mitochondria, cytochrome $\mathrm{c}$ causes the release of an apoptosis-inducing factor, activation of Bcl-2 - associated X protein (BAX), biological downregulation of $\mathrm{B}$-cell lymphoma (Bcl)-2, and release of molecules involved in reactive oxygen species [9]. In addition, p53 consists of $\mathrm{N}$-terminal activation domain, a basic DNA-binding domain with residues within 100-300, and C-terminal basic domain, residues 356-393 [10,11]. The amino acid residues are involved in the activation of p53 in cell cycle arrest that includes DNA repair and apoptosis [12].

Computationally, biological interaction with proteins, resulting in suppression of apoptosis is frequently linked with increased expression of anti-apoptotic proteins and decreased expression of pro-apoptotic proteins [13]. Insight of protein interactions by three-dimensional (3D) structural studies of anti-apoptotic Bcl-2 family [14] reveal an important role in mitochondria pathways-mediated apoptosis; in this, amino acid residues interact with many kinds of small molecules [15], and the overexpression of anti-apoptotic protein is induced [16] in various cancer cells, including colon cancer. Colon cancer leads to mutation [17] in wnt/beta catenin pathway [18] molecules, such as beta catenin and adenomatous polyposis coli (APC). In addition, the APC gene molecular lesions are observed in the majority of familial AP [19] and it regulates a number of cellular functions, which include mitosis, migration, and the maintenance of genome stability [20].

Molecular docking is a computational technique that predicts the noncovalent interaction between two macromolecules or more repeatedly in a macro and small molecules [21]. Drug discovery, molecular docking, and virtual screening, offering multi-user capability, enhanced accuracy, are performed byAutoDockVina; it is anewopen-sourceprogram. Thissoftware was executed by Dr. Oleg Trott in the Molecular Graphics Laboratory (MGL) at the Scripps Research Institute. AutoDock Vina significantly enhances the average accuracy of the binding mode predictions, scoring functions, optimization algorithm, and implementations [22].

This study evaluated interaction of amino acid residues from different types of proteins and root-mean-square deviation (RMSD) differentiation of lower binding and upper bindings of eriodictyol with proteins using the docking score. With this background, this study was estimated in eriodictyol might be acting as colonic tumor suppressor gene like APC and other apoptotic proteins in computational docking studies.

\section{METHODS}

Molecular docking studies

3D crystallographic structures of proteins were obtained from Protein Data Bank (PDB) (http://www.pdb.rcsb.org), and those of 
small molecules were retrieved from PubChem compound database (http://www.ncbi.nlm.nih.gov/search). Molecular docking studies were done using AutoDock Vina, version 1.1.2, of the MGL at the Scripps Research Institute. MGL tool, version 1.5.6, from MGL at the Scripps Research Institute, was used for visualization and analysis of molecular structures. Other software used were Discovery Studio Visualizer 3.5, downloaded from BIOVIA community, Q-site finder (ligand binding site prediction), and PyMOL 1.3 Molecular Graphics System.

\section{Preparation of proteins and ligands}

The 3D structures of proteins were retrieved as follows: p53 from PDB ID: 1 TUP; caspase 8, PDB ID: 2Y1L; Bcl-2, PDB ID: 1MAZ; BAX, PDB ID: 1F16; APC, PDB ID: 1DEB. Initially, DNA, ligand, and crystallographic water molecules were removed in the 3D structure using Discovery Studio Visualizer; AutoDock Tools assigned polar hydrogen, Kollman United atom charges, salvation parameters, and fragmental volumes to the protein. AutoDock saved the prepared protein file in PDBQT format. The 3D structure of eriodictyol saved in SDF format was converted to PDB format, and using MGL tools, hydrogen atoms were added to check the valencies of the heavy atoms. The ligand was minimized by computing Gasteiger charges and saved in PDBQT format.

\section{Grid generation and molecular docking}

The auto grid was used for the preparation of the grid map using a grid box. Points on a 3D grid were placed to cover the complete inner cavity of the receptor that constitutes the ligand. The grid size was set to $40 \times 40 \times 40 \mathrm{xyz}$ points with grid spacing of $0.375 \mathrm{~A}^{\circ}$ and grid center was designed at dimensions ( $\mathrm{x}, \mathrm{y}$, and $\mathrm{z}$ ): 58.176, 15.383, and 78.349 (PDB ID: 1TUP); $-3.42,17.15$, and -31.714 (PDB ID: 2Y1L); 1.936 , 22.227, and 39.275 (PDB ID: 1MAZ); 1.0125, 4.0125, and -1.0125 (PDB ID: 1F16); and 44.052, 7.925, and 3.108 (PDB ID: 1DEB), respectively. A scoring grid was computed using the ligand structure to reduce the computation time. Docking was carried out using AutoDock Vina with protein and ligand data together with the grid box properties in the configuration file. It uses iterated local search global optimizer with the AMBER force field. Throughout the docking studies, the protein molecule was kept as rigid and ligand molecules as flexible.

The results $<2.0 \AA$ in positional RMSD were grouped together, and the outcome with the most favorable free energy of binding was used for representation. The pose with lowest binding energy or binding affinity was used for subsequent analysis.

\section{RESULTS AND DISCUSSION}

\section{Binding affinity}

AutoDock Vina with combined optimization algorithm was performed in molecular docking [23]. Binding energy scoring with AutoDock Vina is generally designed to reduce computing costs and used to estimate

Table 1: Predicted free energy of $\Delta G$ proteins with eriodictyol

\begin{tabular}{llllll}
\hline Ligand & \multicolumn{6}{l}{ Target proteins-G-score (kcal/mol) } \\
\cline { 2 - 6 } Eriodictyol & p53 & Caspase 8 & Bcl-2 & BAX & APC \\
\hline & -10.6 & -10.9 & -9.0 & -9.5 & -7.2 \\
\hline
\end{tabular}

Bcl-2: B-cell lymphoma-2, BAX: Bcl-2 - associated X protein, APC: Adenomatous polyposis coli large numbers of receptors-ligand complexes [24]. In these receptorligand interactions, the prediction of binding energy was evaluated by free energy simulation techniques [25]. We first developed binding energy scoring function of eriodictyol docked with different types of tumor suppressor proteins and apoptotic proteins. The computational analysis of this drug was the first time to understand the mechanism of interaction [26] between eriodictyol and apoptotic proteins. Table 1 shows the values for $\mathrm{p} 53$, caspase $8, \mathrm{Bcl}-2$, BAX, and APC to be $-10.6,-10.9,-9.0,-9.5$, and $-7.2 \mathrm{kcal} / \mathrm{mol}$, respectively. All the proteins were having high affinity and strongly bonded with polar contacts (Fig. 2). The results proved that eriodictyol highly interacted with the colon cancer tumor suppressor gene of APC.

\section{Interaction of amino acid residues}

The interaction linking of amino acids in proteins has been explored for several decades using knowledge-based approaches and amino acids making contact with the hydrophobic interaction into small molecules [27]. During the folding process of amino acids, all water molecules were removed from the functional group of proteins for finding out the binding energy [28], the polar and nonpolar amino acid residues were interacted with eriodictyol. Table 2 shows that p53 contains CYS-277 and ALA-276; caspase 8 contains THR-467, THR-337 (2), and GLU-396 (2); Bcl-2 contains ARG-103 (3), ALA104 (2), PHE-105, and TYR-101; BAX contains GLY-108, TRY-107, ASN106, and GLN-155 (2); APC contains GLU-40 (2) and LEU-37 (2).

\section{RMSD calculations}

RMSD was used for the assessment of docking accuracy of the fraction of protein-ligand contacts [29] and the successful prediction of RMSD $<2 \AA$ [30]. The RMSD prediction was evaluated from the lower and upper binding energies of proteins with eriodictyol. Fig. 3 shows the calculation of RMSD for target proteins of p53, caspase 8, Bcl-2, BAX, and APC, with the lower binding energy of 11.6, 12.5, 15.9, 19.6, and 15.6 and upper binding energies of 12.4, 15.3, 16.9, 20.7, and 18.0, respectively. In AutoDock Vina, we measured 3D structure by RMSD in optimal rigid body superposition. The quantitative comparison between protein and ligand was performed; the predicted structure of folded protein and ligand was also calculated.

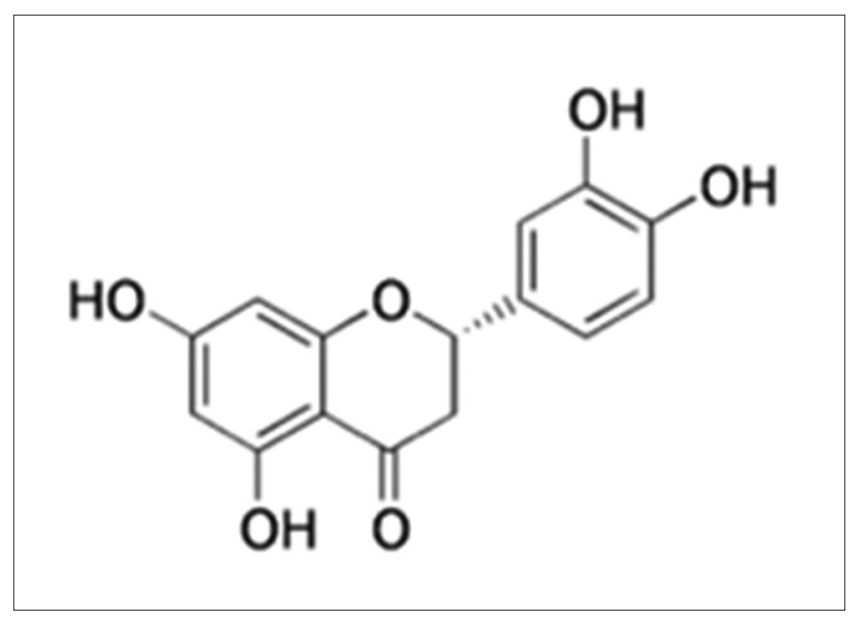

Fig. 1: The molecular structure of eriodictyol

Table 2: Interacted amino acid residues of proteins with eriodictyol

\begin{tabular}{|c|c|c|c|c|c|}
\hline \multirow{2}{*}{$\begin{array}{l}\text { Ligand } \\
\text { Eriodictyol }\end{array}$} & \multicolumn{5}{|c|}{ Target proteins-interacting amino acid residue (s) } \\
\hline & p53 & Caspase 8 & Bcl-2 & BAX & APC \\
\hline & CYS-277 & THR-467 & ARG-103 (3) & GLY-108 & GLU-40 (2) \\
\hline & ALA-276 & THR-337 (2) & ALA-104 (2) & TRY-107 & LEU-37 (2) \\
\hline & & GLU-396 (2) & PHE-105 & ASN-106 & \\
\hline & & & TYR-101 & GLN-155 (2) & \\
\hline
\end{tabular}

Bcl-2: B-cell lymphoma-2, BAX: Bcl-2 - associated X protein, APC: Adenomatous polyposis coli 


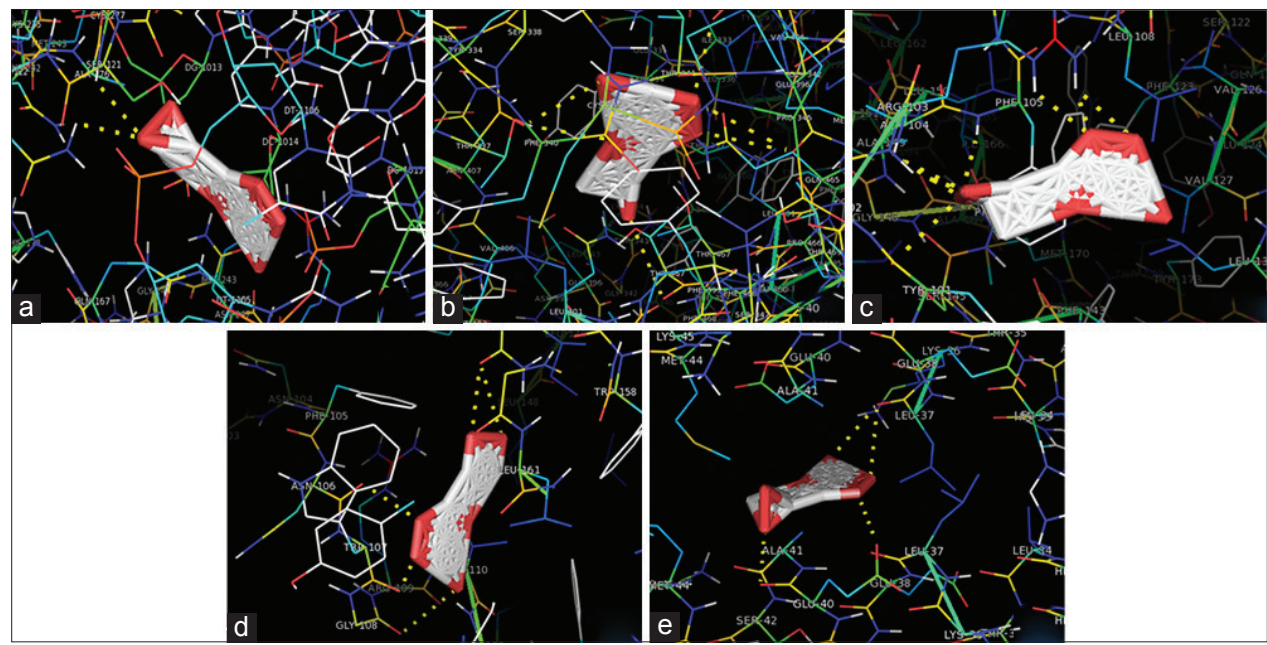

Fig. 2: Structural-based docking of identifying compounds of eriodictyol with proteins. Eriodictyol docked with different types of proteins such as (a) p53, (b) caspase 8, (c) B-cell lymphoma-2, (d) Bcl-2 - associated X protein, and (e) adenomatous polyposis coli. The residues involved in the interaction with the ligand are shown; the hydrogen bonding is indicated in yellow

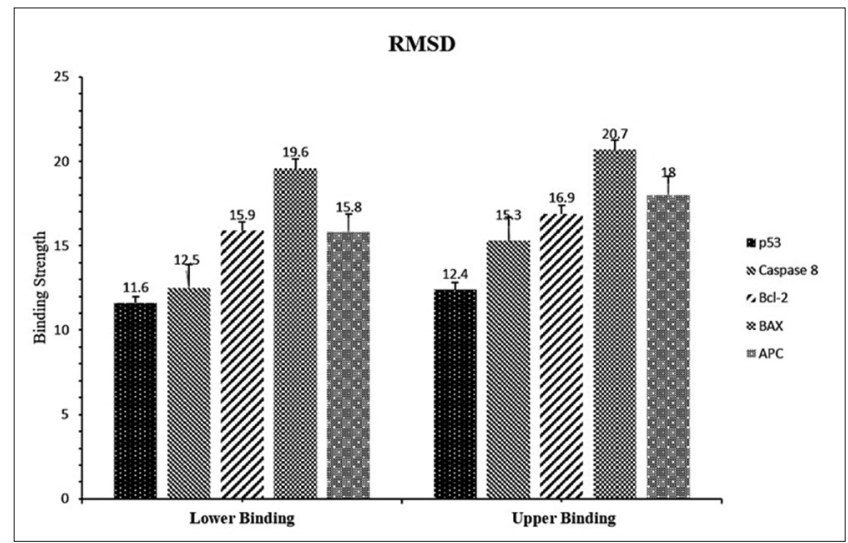

Fig. 3: Root-mean-square deviation difference from lower binding and upper binding energies of eriodictyol with p53, caspase 8, B-cell lymphoma-2 (Bcl-2), Bcl-2 - associated X protein, and adenomatous polyposis coli

\section{CONCLUSION}

Molecular docking was well identified in computational studies using the AutoDock Vina. In this study, we have evaluated the interaction of five macromolecules with the small molecule of eriodictyol, which bounded well and possessed a high score of binding affinity; calculation of RMSD in below $2 \AA$ and the determination of specific amino acid residues that interacted with the small molecule were also identified. The results proved that the small molecules can strongly interact with apoptotic and tumor suppressor genes and binding scores of colonic suppressor proteins showed high affinity when interacted with the small molecule. The novelty of this study was estimated in APC act a tumor suppressor gene in colon cancer, the docking results proved that eriodictyol interacted with five different proteins, but the APC gene having more affinity compared than other genes. In addition to this, with the help of these results, it was proved that eriodictyol also has anti-inflammatory activity in colon cancer. Future studies should estimate the interaction of eriodictyol with colon cancer apoptotic pathways in both in vitro and in vivo.

\section{REFERENCES}

1. Karthi N, Manju V. Synergistic effects of selected anticancer drugs with vitamin-E against HT-29 human adenocarcinoma cell lines. Indian J Res Pharm Biotechnol 2014;2(1):1246-53.
2. Cancer Facts and Figures. The American Cancer Society, 2016. Available from: http://www.cancer.org.

3. Coker-Gurkan A, Arisan ED, Obakan P, Guvenir E, Unsal NP. Inhibition of autophagy by 3-MA potentiates purvalanol-induced apoptosis in Bax deficient HCT 116 colon cancer cells. Exp Cell Res 2014;328(1):87-98

4. Bucolo C, Leggio GM, Drago F, Salomone S. Eriodictyol prevents early retinal and plasma abnormalities in streptozotocin-induced diabetic rats. Biochem Pharmacol 2012;84(1):88-92.

5. Hu Q, Zhang DD, Wang L, Lou H, Ren D. Eriodictyol-7-O-glucoside, a novel Nrf2 activator, confers protection against cisplatin-induced toxicity. Food Chem Toxicol 2012;50(6):1927-32.

6. Huang TC, Tseng KY, Tsai SS, Liu HJ, Ho CH, Lin HY, et al. Eriodictyol decreases very late antigen-4 (VLA-4) expression, cellular adhesion, and migration through an NF kB-dependent pathway in monocytes. J Funct Foods 2012;2:263-70.

7. Imen MB, Chaabane F, Nadia M, Soumaya KJ, Kamel G, Leila CG. Anti-melanogenesis and antigenotoxic activities of eriodictyol in murine melanoma (B16-F10) and primary human keratinocyte cells. Life Sci 2015;135:173-8.

8. Lou H, Jing X, Ren D, Wei X, Zhang X. Eriodictyol protects against $\mathrm{H}(2) \mathrm{O}(2)$-induced neuron-like $\mathrm{PC} 12$ cell death through activation of Nrf2/ARE signaling pathway. Neurochem Int 2012;61(2):251-7.

9. Kim MJ, Yun HS, Hong EH, Lee SJ, Baek JH, Lee CW, et al. Depletion of end-binding protein 1 (EB1) promotes apoptosis of human non-small-cell lung cancer cells via reactive oxygen species and Bax-mediated mitochondrial dysfunction. Cancer Lett 2013;339(1):15-24.

10. Harms KL, Chen $\mathrm{X}$. The $\mathrm{C}$ terminus of $\mathrm{p} 53$ family proteins is a cell fate determinant. Mol Cell Biol 2005;25(5):2014-30.

11. De Grandis V, Bizzarri AR, Cannistraro S. Docking study and free energy simulation of the complex between p53 DNA-binding domain and azurin. J Mol Recognit 2007;20(4):215-26.

12. Janouskova H, Ray AM, Noulet F, Lelong-Rebel I, Choulier L, Schaffner F, et al. Activation of $\mathrm{p} 53$ pathway by Nutlin-3a inhibits the expression of the therapeutic target a5 integrin in colon cancer cells. Cancer Lett 2013;336(2):307-18.

13. Jourdan M, Reme T, Goldschmidt H, Fiol G, Pantesco V, De Vos J, et al. Gene expression of anti - And pro-apoptotic proteins in malignant and normal plasma cells. Br J Haematol 2009;145(1):45-58.

14. Shipra G, Gauri M, Chandra PM, Kishore SP. Identification of novel potent inhibitors against Bcl-xL anti-apoptotic protein using docking studies. Protein Pept Lett 2012;19(12):1302-17.

15. Li SZ, Zhang HH, Zhang JN, Zhang ZY, Zhang XF, Zhang XD, et al. ALLN hinders HCT116 tumor growth through Bax-dependent apoptosis. Biochem Biophys Res Commun 2013;437(2):325-30.

16. Hu T, Wang L, Zhang L, Lu L, Shen J, Chan RL, et al. Sensitivity of apoptosis-resistant colon cancer cells to tanshinones is mediated by autophagic cell death and p53-independent cytotoxicity. Phytomedicine 2015;22(5):536-44.

17. Dong GZ, Lee SY, Zhao HY, Lee YI, Jeong JH, Jeon R, et al. 
Diarylheptanoids from lesser galangal suppress human colon cancer cell growth through modulating Wnt/ $\beta$-catenin pathway. J Funct Foods 2015;18:47-57.

18. Cristofaro M, Contursi A, D'Amore S, Martelli N, Spaziante AF, Moschetta A, et al. Adenomatous polyposis coli (APC)-induced apoptosis of HT29 colorectal cancer cells depends on mitochondrial oxidative metabolism. Biochim Biophys Acta 2015;1852(9):1719-28.

19. Kim JH, Kim YH, Song GY, Kim DE, Jeong YJ, Liu KH, et al. Ursolic acid and its natural derivative corosolic acid suppress the proliferation of APC-mutated colon cancer cells through promotion of $\beta$-catenin degradation. Food Chem Toxicol 2014;67:87-95.

20. Dow LE, O'Rourke KP, Simon J, Tschaharganeh DF, van Es JH, Clevers $\mathrm{H}$, et al. Apc restoration promotes cellular differentiation and reestablishes crypt homeostasis in colorectal cancer. Cell 2015;161(7):1539-52.

21. Lengauer T, Rarey M. Computational methods for biomolecular docking. Curr Opin Struct Biol 1996;6(3):402-6.

22. Trott O, Olson AJ. AutoDock Vina: Improving the speed and accuracy of docking with a new scoring function, efficient optimization, and multithreading. J Comput Chem 2010;31(2):455-61

23. Jung IP, Cho JH, Koo BS, Yoon MY. Functional evaluation of residues in the herbicide-binding site of Mycobacterium tuberculosis acetohydroxyacid synthase by site-directed mutagenesis. Enzyme Microb Technol 2015;78:18-26.

24. Ren W, Truong TM, Ai HW. Study of the binding energies between unnatural amino acids and engineered orthogonal tyrosyl-tRNA synthetases. Sci Rep 2015;5:12632.

25. Kitchen DB, Decornez H, Furr JR, Bajorath J. Docking and scoring in virtual screening for drug discovery: Methods and applications. Nat Rev Drug Discov 2004;3(11):935-49.

26. Ranjan S, Dasgupta N, Chinnappan S, Ramalingam C, Kumar A. A novel approach to evaluate titanium dioxidenanoparticle-protein interaction through docking: An insight into mechanism of action. Proc Natl Acad Sci India B Biol Sci 2015;1-7. DOI: 10.1007/s40011-015-0673-Z.

27. Jha AN, Vishveshwara S, Banavar JR. Amino acid interaction preferences in proteins. Protein Sci 2010;19(3):603-16.

28. Kyte J, Doolittle RF. A simple method for displaying the hydropathic character of a protein. J Mol Biol 1982;157(1):105-32

29. Feinstein WP, Brylinski M. Calculating an optimal box size for ligand docking and virtual screening against experimental and predicted binding pockets. J Cheminform 2015;7:18.

30. Alhossary A, Handoko SD, Mu Y, Kwoh CK. Fast, accurate, and reliable molecular docking with QuickVina 2. Bioinformatics 2015;31(13):2214-6. 